\title{
Indirect Evidence for Self Association of Muscle Fatty Acid Binding Protein from Locusta migratoria
}

\author{
Abdul Hai, Nadeem A. Kizilbash, Syeda Huma H. Zaidi, and Jamal Alruwaili
}

\begin{abstract}
Fatty acid binding proteins are involved in the storage and transport of long chain fatty acids. This study about the muscle fatty acid binding protein of Locusta migratoria (LM-FABP) was pursued by the use of far-UV Circular Dichroism (CD) spectroscopy. It provides indirect evidence that the protein self-associates in solution. A hydrophobic patch on the surface of LM-FABP, reported earlier, can mediate self-association between the protein monomers. The property of self-association can also affect the activity of membrane-bound enzymes in locusts such as Adenine Nucleotide Translocase (also known as the ADP/ATP translocator) which is fatty acid or acyl coenzymeA dependent.
\end{abstract}

Index Terms - Circular dichroism, muscle fatty acid binding protein, locusta migratoria, self-association.

\section{INTRODUCTION}

Fatty acid-binding proteins (FABPs) comprise a family of carrier proteins for fatty acids, eicosanoids and retinoids [1], [2]. These proteins facilitate the transfer of fatty acids between extra- and intracellular membranes [3]. Some family members also transport lipophilic molecules from outer cell membrane to certain intracellular receptors such as PPAR [4]. The role of fatty acid binding proteins (FABPs) in lipid metabolism is well known [5], [6]. The major fuel driving the performance of the muscle cells, in Locusta migratoria, is provided by the long chain fatty acids during extended flights. A metabolic system operates in the muscles, for moving fatty acids across successive hydrophobic and hydrophilic barriers to the mitochondrial matrix. The exact mechanism of operation of this system requires further investigation. The generally accepted consensus is that fatty acids, before $\beta$-oxidation in the mitochondrial matrix, are first transformed into acyl-CoA in the outer mitochondrial membrane, then into acylcarnitine on the external surface of the inner membrane. The fatty acids are moved across the inner mitochondrial membrane and then retransformed into acyl-CoA on the matrix side of the inner membrane. Finally, acyl-CoA is $\beta$-oxidized in the mitochondrial matrix.

The property of self-association or aggregation is one of the characteristic properties of many native and non-native

Manuscript received March 2, 2014; revised May 12, 2014.

Abdul Hai and Nadeem A. Kizilbash are with the Department of Biochemistry, Faculty of Medicine \& Applied Medical Sciences, Northern Border University, Arar, Saudi Arabia (e-mail: synavia@gmail.com, fsd707@ gmail.com).

Syeda Huma H. Zaidi is with the Department of Chemistry, Faculty of Science, Northern Border University, Arar, Saudi Arabia (e-mail: humazaidi@gmail.com).

Jamal Alruwaili is with the Faculty of Medicine \& Applied Medical Sciences, Northern Border University, Arar, Saudi Arabia (e-mail: malkh1195@hotmail.com). proteins. The self-association or aggregation of proteins results in the development of such diseases as Down's syndrome, Alzheimer's disease [7], cataracts [8], [9] and many others. The formation of inclusion bodies is a major problem in the overexpression of recombinant proteins [10]-[13], while the production and in vivo delivery of protein drugs is often complicated by association [14].

Adenine Nucleotide Translocase (ANT) is an integral protein of the inner mitochondrial membrane that plays an important role in cellular bioenergetics [15], apoptosis [16] and cell signaling [17]. ANT catalyzes the exchange of ADP for ATP across the inner mitochondrial membrane thereby coupling oxidative phosphorylation to cell metabolism. The property of self-association of muscle FABP of Locusta migratoria was investigated by Circular Dichroism (CD) spectroscopy. Self-association of LM-FABP can produce an effect on the activity of membrane-bound enzymes in insects such as Adenine Nucleotide Translocase (also known as the ADP/ATP translocator) which is fatty acid or acyl coenzymeA dependent.

\section{MATERIALS AND METHODS}

LM-FABP was purified by the use of previously published protocols (Maatman et al., 1994). The protocol included cell lysis, centrifugation and purification by a Sepharose HR 26/10 FPLC column. LM-FABP eluted with $50 \mathrm{mM}$ Tris/ $\mathrm{HCl}$ $(\mathrm{pH}$ 8.0). The fractions were analyzed by SDS/PAGE and Western blotting. Fractions containing LM-FABP were further purified by gel filtration chromatography using a Sephacryl S-100 column. For these experiments, LM-FABP sample in $20 \mathrm{mM}$ phosphate buffer ( $\mathrm{pH}$ 5.42) was used. The concentration of the sample was determined by measuring the $\mathrm{UV}$-Vis absorbance at $280 \mathrm{~nm}$. The absorbance of light at 280 $\mathrm{nm}$ provides an estimate of the concentration of the protein sample (LM-FABP has seven Phenylalanines and three Tyrosines; the absorbance by each of these is estimated to contribute about 1280 O.D. units to the total absorbance).

Far-UV CD spectra (190-250 nm) were measured by an Aviv Associates model 62DS spectrometer (Aviv Associates, Lakewood, NJ) equipped with a temperature controlling device and calibrated with Camphorosulfonic acid. The CD spectra were acquired by using sample concentrations of 4.7, $16,18.8$ and $100 \mu \mathrm{g} / \mathrm{mL}$ at $35^{\circ} \mathrm{C}$. A thermal melt experiment was also performed by using a sample concentration of 18.8 $\mu \mathrm{g} / \mathrm{mL}$ (Fig. 1). The irreversibility of the unfolding process was established by cooling the sample to $35{ }^{\circ} \mathrm{C}$ and re-measuring the $\mathrm{CD}$ spectrum. The measured thermal transition had a midpoint of $\approx 70{ }^{\circ} \mathrm{C}$ and unfolding of the protein was not reversible (Fig. 2 and Fig. 3). 


\section{RESULTS}

The Circular Dichroism spectra of LM-FABP are characterized by a minimum at $218 \mathrm{~nm}$ (Fig. 1). This is typical for a protein rich in $\beta$-secondary structure. The ellipticity value at $218 \mathrm{~nm}$ was chosen as the parameter describing the global secondary structure variations of the protein. The ellipiticity decreased between 202 and $233 \mathrm{~nm}$ with the dilution of the protein sample. This can be taken as the indirect evidence that oligomeric species formed in solution. These concentration-dependent variations can be understood if we assume interactions of aggregation-type between the protein molecules. Between 202 and $233 \mathrm{~nm}$, the CD spectra show a maximum and a minimum (Fig. 1). For the $4.7 \mu \mathrm{g} / \mathrm{mL}$ concentration protein sample, a considerable reduction of signal was found. The ellipiticity values are not sensitive to variations of concentration when the protein is monomeric, but are expected to vary if the protein molecules collide and then self-aggregate; molecular conformational changes are required for this process.

A thermal melt experiment (Fig. 2) provided melting point $\left(T_{m}\right)$ of LM-FABP in solution. The CD spectra were compared for the protein in the folded state and the same protein sample re-folded back after denaturation (Fig. 3). The CD spectra showed that this protein unfolds by a single-step mechanism and the unfolding is irreversible.

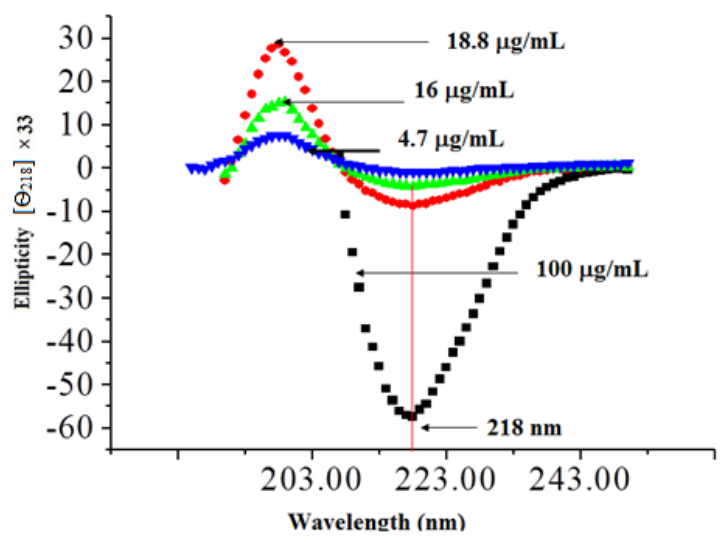

Fig. 1. Superimposed CD spectra of LM-FABP showing the concentration-dependent change in ellipticity.

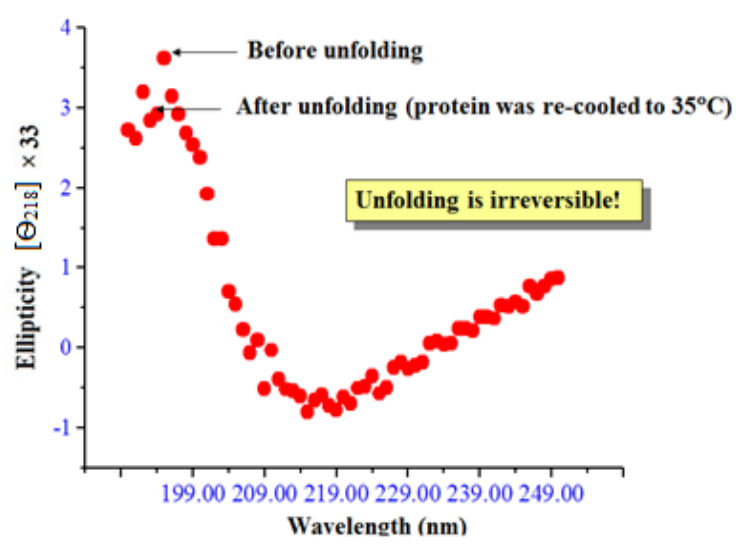

Fig. 2. Superimposed CD spectra showing the irreversibility of the unfolding process.

The first $\mathrm{CD}$ spectrum was acquired at $35^{\circ} \mathrm{C}$ of the protein in the folded state. The second $\mathrm{CD}$ spectrum was acquired after heat denaturation of the protein and then cooling to 35 ${ }^{\circ} \mathrm{C}$.

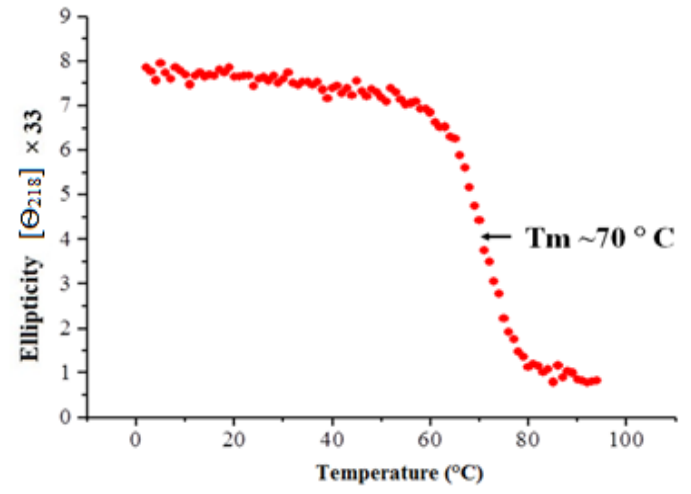

Fig. 3. Thermal melt experiment showing that the melting point $\left(\mathrm{T}_{\mathrm{m}}\right)$ of $\mathrm{LM}-\mathrm{FABP}$ is $70^{\circ} \mathrm{C}$ in solution

\section{DISCUSSION}

Interactions between proteins are important for many biological functions such as signal transduction involves protein-protein interactions. Signal transduction plays an important role in many biological processes and diseases such as cancer. Proteins might interact for a long time to form part of a protein complex or just briefly with another protein to modify it. The modification of proteins can itself change protein-protein interactions.

Protein-protein interactions [18] involve both self-association and aggregation. Self-association of proteins [19] is different from aggregation because the latter involves formation of a disulfide bond. But in self-association, oligomeric species can form simply by hydrophobic interactions alone [20]. After aggregation, the species typically does not remain solubilized but precipitates out of solution. The aggregation property of the fatty acid-carrier protein appears to be an efficient parameter to be considered in the control of the activity of membrane-bound enzymes depending upon the availability of fatty acid or acyl-CoA substrates. This control might concern the major metabolic pathway generating energy in muscle cells i.e. by the mitochondrial $\beta$-oxidative system.

Circular dichroism (CD) refers to the differential absorption of left and right circularly polarized light. The far-UV CD spectrum of proteins can reveal information about the secondary as well as the quaternary structure of the protein. CD spectra can be readily used to estimate the fraction of a molecule that is in the $\alpha$-helix, $\beta$-strand or the $\beta$-turn conformation, or investigate the presence of self-association among protein molecules [21], [22]. CD, however, cannot, in general, say where the $\alpha$-helices or $\beta$-strands are located within the protein molecule or even completely predict how many there are. $\mathrm{CD}$ gives less specific structural information than X-ray crystallography and protein NMR spectroscopy, for example, which both give atomic resolution data.

Hydrophobicity determines the structure and function of proteins. It is responsible for the folding of soluble proteins [23]. The energetic cost of exposing a hydrophobic surface to water is proportional to its area. Self-association can be driven by hydrophobic patches on the protein surface. It involves loss of entropy for the monomers or subunits. In a dimeric complex, the negative entropy change arises due to the loss of six motional degrees of freedom and additional 
losses in mobility of interface side-chains. The entropic cost of dimerization has been reported at 20-30 kcal/mol [24]. A hydrophobic patch was reported earlier on the surface of LM-FABP comprising Ile45, Phen51, Phen64 and Phen66 [25] (Fig. 4). This hydrophobic patch can mediate the observed self-association of LM-FABP being reported in this study.

A hydrophobic patch on a protein surface ranges from 200 to 1,200 amino acids and averages around 400 amino acids [27]. The hydrophobic patches are not related to the sizes of the proteins and only weakly to their non-polar surface fraction [28]. Ala, Lys, and Pro provide the highest contributions to non-polar surface fraction for smaller patches [29]. The contribution of the hydrophobic amino acids becomes more important as the patch size increases. The polar amino acids expose an approximately constant fraction of their non-polar area independent of patch size [30]. Though the mobility of residues on the surface is generally higher, it decreases for the polar residues with increasing patch size [31].

Thermal stability of a protein is assessed using CD by following changes in the spectrum with increasing temperature [32]. Sometimes, the entire spectrum in the faror near-UV CD region can be followed at a number of temperatures. Or, a single wavelength can be chosen which monitors some specific features of the protein structure. The signal at that wavelength is then recorded continuously as the temperature is raised. $\mathrm{CD}$ is often used to investigate the effect of solution $\mathrm{pH}$, buffers, and additives such as sugars, amino acids or salts on thermal stability [33]. In this study, the ellipticity value at $218 \mathrm{~nm}$ was chosen as the parameter describing the global secondary structure variations of the protein. The ellipiticity decreased between 202 and $233 \mathrm{~nm}$ with the dilution of the protein sample (Fig. 1). This can be taken as the indirect evidence that oligomeric species formed in solution.

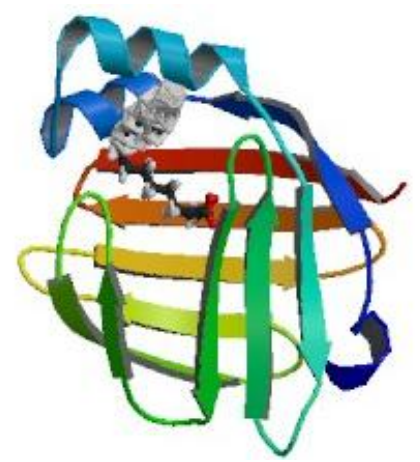

Fig. 4. Fatty acid binding protein from locust flight muscle in complex with Oleic acid [26].

Many proteins aggregate or precipitate after they are unfolded, making the process of unfolding irreversible [34]. The reversibility of the unfolding reaction can be assessed by cooling the sample and then heating again to see if the unfolding reaction is duplicated. If the melting is reversible, the melting temperature $\left(T_{m}\right)$ is proportional to conformational stability and the thermodynamics of protein folding can be extracted from the data [34]. The two superimposed $C D$ spectra shown in Fig. 2 represent the protein in the folded state and the same protein sample re-folded back after denaturation. These spectra showed that LM-FABP unfolds by a single-step mechanism and the unfolding is irreversible.

\section{CONCLUSION}

The central theme of this study is about the property of self-association of muscle fatty acid binding protein of Locusta migratoria. Experimental evidence has been acquired by use of a simple variable, i.e. the probability of intermolecular encounters controlled by changes in protein concentration. The experimental data shows that fluctuations of ellipticity take place in CD spectra which suggest that the structural changes take place at the same time as the self-association process in the external areas of the protein molecule.

\section{REFERENCES}

[1] A. Chmurzyńska, "The multigene family of fatty acid-binding proteins (FABPs): function, structure and polymorphism," J. Appl. Genet., vol. 47, no. 1, pp. 39-48, 2006.

[2] R. L. Smathers and D. R. Petersen, "The human fatty acid-binding protein family: Evolutionary divergences and functions," Hum Genomics, vol. 5, no. 1, pp. 170-191, 2011.

[3] R. A. Weisiger, "Cytosolic fatty acid binding proteins catalyze two distinct steps in intracellular transport of their ligands," Mol. Cell. Biochem., vol. 239, no. 1-2, pp. 35-43, 2002.

[4] N. S. Tan, N. S. Shaw et al., "Selective cooperation between fatty acid binding proteins and peroxisome proliferator-activated receptors in regulating transcription," Mol. Cell. Biol., vol. 22, no. 14, pp. 5114-27, 2002.

[5] N. Bass, "The cellular fatty acid binding proteins: aspects of structure, function and regulation," International Review of Cytology, vol. 111, pp. 143-185, 1988.

[6] F. Spener, T. Borchers, and M. Mukhejea, "On the role of fatty acidbinding protein in fatty acid transport and metabolism," FEBS Letters, vol. 244, pp. 1-5.

[7] S. Massry and R. Glassock, Textbook of Nephrology, Williams and Wilkins, Baltimore, 1983.

[8] J. Clark and J. Steele, "Phase-separation inhibitors and prevention of selenite catarac," Natl. Acad. Sci., vol. 89, pp. 1720-1722, 1992.

[9] J. T hompson, P. Schurtenberger et al., "Binary liquid phase separation and critical phenomena in a protein/water solution," Natl. Acad. Sci. USA. vol. 84, pp. 7079 -7082, 1987.

[10] F. Marston, "The purification of eukaryotic polypeptides synthesized in Escherichia coli," Biochem. J., vol. 240, pp. 1-12, 1986.

[11] C. Schein, "Solubility as a function of protein structure and solvent components," Biotechnology, vol. 7, pp. 1141-1149, 1989.

[12] R. Wetzel, "Principles of protein stability. Part 2. Enhanced folding and stabilization of proteins by suppression of aggregation in vitro and in vivo," in Protein Engineering: A Practical Approach, A. R. Rees, A. R. Sternberg, and R. Wetzel, Eds. Oxford, IRL Press, 1992, pp. 191-219.

[13] R. Wetzel, "Protein aggregation in vivo: Bacterial inclusion bodies and mammalian amyloid," in Stability of Protein Pharmaceuticals, Part B: In Vivo Pathways of Degradation and Strategies for Protein Stabilization, T. J. Ahern and M. C. Manning, Eds. New York, Plenum Press, 1992, vol. 3, pp. 43-88.

[14] W. Lougheed et al., "Insulin aggregation in artificial delivery systems," Diabetol., vol. 19, pp. 1-8, 1980.

[15] E. Pebay-Peyroula and G. Brandolin, "Nucleotide exchange in mitochondria: Insight at a molecular level," Current Opinion in Structural Biology, vol. 14, no. 4, pp. 420-425, 2004.

[16] A. Dorner and H. Schultheiss, "Adenine nucleotide translocase in the focus of cardiovascular diseases," Trends in Cardiovascular Med., vol. 17, no. 8, pp. 284-290, 2007.

[17] J. Sharer et al., "ARL2 and BART enter mitochondria and bind the adenine nucleotide transporter," Molecular Biology of the Cell, vol. 13, no. 1, pp. 71-83, 2002.

[18] J. L. Oncley et al., "Protein-protein interactions," The Journal of Physical Chem., vol. 56, no. 1, pp. 85-92, 1952. 
[19] P. D. Ross and S. Subramanian, "Thermodynamics of protein association reactions: forces contributing to stability," Biochem., vol. 20, no. 11, pp. 3096-3102, 1981.

[20] E. Diego et al., "H-NS oligomerization domain structure reveals the mechanism for high order self-association of the intact protein," Journal of Mol. Biol., vol. 324, no. 4, pp. 841-850, 2002.

[21] L. Whitmore and B. A. Wallace, "Protein secondary structure analyses from circular dichroism spectroscopy: methods and reference databases," Biopolym., vol. 89, no. 5, pp. 392-400, 2008.

[22] N. Greenfield, "Using circular dichroism spectra to estimate protein secondary structure," Nature Protocols, vol. 1, no. 6, pp. 2876-90, 2006.

[23] K. A. Dill, "Theory for the folding and stability of globular proteins," Biochem., vol. 24, no. 6, pp. 1501-1509, 1985.

[24] J. Janin, S. Miller, and C. Chothia, "Surface, subunit interfaces and interior of oligomeric proteins," J. Mol. Biol., vol. 204, pp. 155-164, 1988.

[25] N. Kizilbash, "Fatty acid binding proteins and hydrophobic ligands," Lambert, Saarbrücken, 2013.

[26] C. Lücke, Y. Qiao et al., "Fatty-acid-binding protein from the flight muscle of Locusta Migratoria: Evolutionary variations in fatty acid binding," Biochem., vol. 45, pp. 6296-6305, 2006.

[27] P. Lijnzaad, H. J. Berendsen, and P. Argos, "Hydrophobic patches on the surfaces of protein structures," Proteins: Structure, Function, and Bioinformatics, vol. 25, no. 3, pp. 389-397, 1996.

[28] R. P. Bahadur, P. Chakrabarti, F. Rodier, and J. Janin, "Dissecting subunit interfaces in homodimeric proteins," Proteins: Structure, Function, and Bioinform., vol. 53, no. 3, pp. 708-719, 2003.

[29] C. J. Tsai, S. L. Lin, H. J. Wolfson, and R. Nussinov, "Studies of protein-protein interfaces: A statistical analysis of the hydrophobic effect," Protein Sci., vol. 6, no. 1, pp. 53-64, 1997.

[30] H. Acharya, S. Vembanur, S. N. Jamadagni, and S. Garde, "Mapping hydrophobicity at the nanoscale: Applications to heterogeneous surfaces and proteins," Faraday Discuss, vol. 146, pp. 353-365, 2010.

[31] P. Lijnzaad, H. J. Berendsen, and P. Argos, "Hydrophobic patches on the surfaces of protein structures," Proteins: Structure, Function, and Bioinform., vol. 25, no. 3, pp. 389-397, 1996.

[32] G. Dantas et al., "A large scale test of computational protein design: folding and stability of nine completely redesigned globular proteins," Journal of Mol. Biol., vol. 332, no. 2, pp. 449-460, 2003.

[33] B. Ranjbar and P. Gill, "Circular dichroism techniques: Biomolecular and nanostructural analyses - A review," Chemical Biol. Drug Design, vol. 74, no. 2, pp. 101-120, 2009.

[34] E. Sahin, A. O. Grillo, M. D. Perkins, and C. J. Roberts, "Comparative effects of $\mathrm{pH}$ and ionic strength on protein-protein interactions, unfolding, and aggregation for IgG1 antibodies," Journal of Pharmaceutical Sci., vol. 99, no. 12, pp. 4830-4848, 2010.

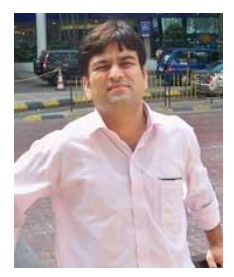

Abdul Hai was born in Allahabad, India. He received his master's degree from University of Allahabad, India. His doctoral degree was awarded in biochemistry in 2002 from IIT-Kanpur in India. He is presently working as an assistant professor of biochemistry at Northern Border University in Arar, Saudi Arabia. He has previously worked as an assistant professor at Garyounis University in Libya. His work experience also includes postdoctoral fellowship at Max Planck (Germany) and University of North Carolina (Raleigh, U.S.A.). He has published eight papers in international journals on topics as diverse as proteomics, protein structure, drug delivery and molecular docking.

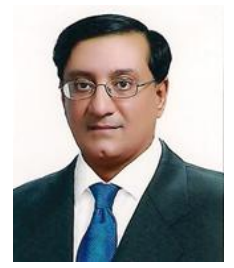

Nadeem Kizilbash was born in Karachi, Pakistan. He received his bachelor's degree in chemistry in 1989 from Longwood College in Farmville, U.S.A. and received his master's degree in chemistry in 1994 from Washington University in St. Louis, U.S.A. His doctoral degree was awarded in biophysics in 2003 from Boston University in U.S.A. He is presently working as an assistant professor of biochemistry at Northern Border University in Arar, Saudi Arabia. He has previously worked as an assistant professor at Quaid-i-Azam University in Pakistan. His work experience also includes service as a senior scientist at National Institute for Biotechnology and Genetic Engineering in Pakistan. He has published fifteen papers in various journals on topics as diverse as proteomics, protein structure, drug delivery and gene polymorphism. Dr. Kizilbash is currently a member of editorial board of three international research journals.

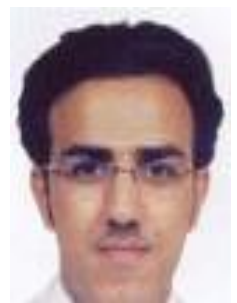

Jamal Alruwaili was born in Arar, Saudi Arabia. He received his doctoral degree in biochemistry in 2011 from the University of Portsmouth in U.K. The topic of his dissertation was: "Serum Proteomic Analysis of Prostate Cancer Progression". He is presently working as the vice dean of the Faculty of Applied Medical Sciences at Northern Border University in Arar, Saudi Arabia. He has previously worked as a scientist at King Faisal Specialist Hospital in Riyadh, Saudi Arabia. He has published papers in international journals on topics as diverse as proteomics, protein structure, molecular docking and drug delivery. 